\title{
Patterns of recurrence after radiation therapy for high-risk neuroblastoma
}

\author{
Ji Hwan Jo, Seung Do Ahn, Minji Koh, Jong Hoon Kim, Sang-wook Lee, Si Yeol Song, \\ Sang Min Yoon, Young Seok Kim, Su Ssan Kim, Jin-hong Park, Jinhong Jung, Eun Kyung Choi \\ Department of Radiation Oncology, Asan Medical Center, University of Ulsan College of Medicine, Seoul, Korea
}

Purpose: To investigate the patterns of recurrence in patients with neuroblastoma treated with radiation therapy to the primary tumor site.

Materials and Methods: We retrospectively analyzed patients with high-risk neuroblastoma managed with definitive treatment with radiation therapy to the primary tumor site between January 2003 and June 2017. These patients underwent three-dimensional conformal radiation therapy or intensity-modulated radiation therapy. A total of 14-36 Gy was delivered to the planning target volume, which included the primary tumor bed and the selected metastatic site. The disease stage was determined according to the International Neuroblastoma Staging System (INSS). We evaluated the recurrence pattern (i.e., local or systemic), progression-free survival, and overall survival.

Results: A total of 40 patients with high-risk neuroblastoma were included in this study. The median patient age was 4 years (range, 1 to 11 years). Thirty patients (75\%) had INSS stage 4 neuroblastoma. At the median follow-up of 58 months, there were 6 cases of local recurrence and 10 cases of systemic recurrence. Among the 6 local failure cases, 4 relapsed adjacent to the radiation field. The other 2 relapsed in the radiation field (i.e., para-aortic and retroperitoneal areas). The main sites of distant metastasis were the bone, lymph nodes, and bone marrow. The 5 -year progression-free survival was $70.9 \%$ and the 5 -year overall survival was $74.3 \%$. Conclusion: Radiation therapy directed at the primary tumor site provides good local control. It seems to be adequate for disease control in patients with high-risk neuroblastoma after chemotherapy and surgical resection.

Keywords: Neuroblastoma, Neoplasm recurrence, Survival, Progression-free survival, Radiotherapy, Retrospective studies

\section{Introduction}

Neuroblastoma is the second most common solid tumor in childhood and is the most common extracranial solid tumor in children. Approximately $90 \%$ of cases occur in children $<10$ years of age, and this disease accounts for about $15 \%$ of the overall childhood cancer mortality [1-3].
Patients with high-risk neuroblastoma, which require multimodality treatment, account for approximately 50\% of all patients with neuroblastoma. These patients are treated with chemotherapy, surgical resection, autologous stemcell transplantation, and radiation therapy (RT). However, the treatment results of these patients have not been satisfactory.

RT is usually applied to the primary tumor site or

Received 26 June 2019, Revised 20 August 2019, Accepted 16 September 2019.

Correspondence: Seung Do Ahn, Department of Radiation Oncology, Asan Medical Center, University of Ulsan College of Medicine, 88 Olympic-ro 43-gil, Songpa-gu, Seoul 05505, Korea. Tel: +82-2-3010-4436, Fax: +82-2-3010-6950, E-mail: sdahn@amc.seoul.kr (http://orcid.org/0000-0003-4153-4191)

(c) This is an Open Access article distributed under the terms of the Creative Commons Attribution Non-Commercial License (http://creativecommons.org/ licenses/by-nc/4.0/) which permits unrestricted non-commercial use, distribution, and reproduction in any medium, provided the original work is properly cited.

www.e-roj.org 
surgical bed after the patient has recovered from stem-cell transplantation. Several studies have shown that RT to the primary tumor site improves the local control rate [4-10].

At the time of the initial diagnosis, approximately $50 \%$ of patients have metastatic disease and distant recurrence is a major obstacle to the treatment of these patients. However, the patterns of recurrence have rarely been reported. Therefore, we investigated the patterns of recurrence in patients with neuroblastoma treated with RT to the primary tumor site.

\section{Materials and Methods}

We retrospectively analyzed patients with high-risk neuroblastoma managed with definitive treatment (i.e., chemotherapy, surgical resection, or stem-cell transplantation) with RT to the primary tumor site between January 2003 and June 2017.

High-risk neuroblastoma was defined according to the Children's Oncology Group risk stratification system [11]. The disease stage was determined using the International Neuroblastoma Staging System (INSS). Computed tomography (CT), whole-body magnetic resonance imaging (WB-MRI; $n$ $=39,97.5 \%$ ), iodine-123 (123I) meta-iodobenzylguanidine single-photon emission computed tomography (MIBG SPECT; $n=27,67.5 \%$ ), bone scan, bone marrow aspiration, and biopsy were performed for staging.

Most of the patients underwent chemotherapy according to the COGANBLOOP1 study [12] or the CCG3891 study [13]. The treatment response of the patients was evaluated using WB-MRI, CT, bone scan, 123I-MIBG SPECT, and bone marrow aspiration.

After induction chemotherapy, surgical resection, and stemcell transplantation, RT was delivered to the primary tumor sites or residual tumor. In some patients, RT was concurrently administered to the metastatic site. Patients who received RT with a palliative intent and those who did not receive $\mathrm{RT}$ to the primary tumor site were excluded from the current study. The gross target volume consisted of the post-induction chemotherapy, pre-surgical tumor volume. The clinical target volume consisted of the primary tumor bed and regional lymph nodes. The planning target volume was set by expanding the clinical target volume by $0.5-1 \mathrm{~cm}$. The treatment of metastatic disease was different for each patient; however, we usually included the gross tumor and the surrounding 1-2 cm (Fig. 1). A CT-based planning was used for treatment planning. The patients were treated with three-dimensional conformal RT or intensity-modulated RT. A total of 14-36 Gy in 7-20

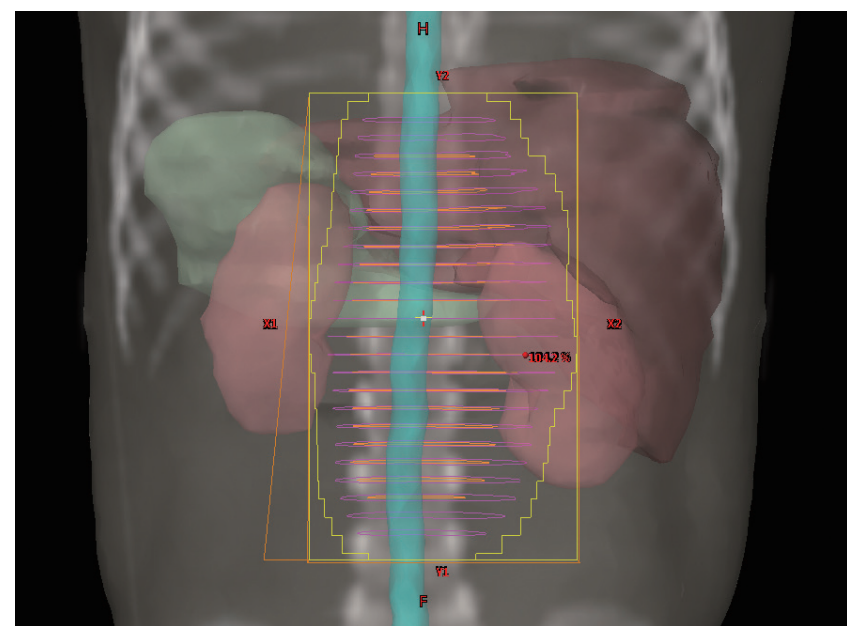

Fig. 1. Radiation therapy treatment field for a patient with a left adrenal primary neuroblastoma who underwent left adrenalectomy.

fractions was delivered to the planning target volume, which included the primary tumor bed and the selected metastatic site (the same dose of radiation was delivered to the primary site and the metastatic site).

We evaluated the recurrence pattern (i.e., local or systemic), progression-free survival (PFS), and overall survival (OS). Local recurrence was defined as disease recurrence in the primary tumor site or regional lymph nodes. Further, we defined adjacent-field failure as recurrence or progression of disease at sites outside the planning target volume that received $<95 \%$ and $>5 \%$ of the prescribed dose. Local failure included both adjacent-field failure and in-field recurrence. PFS was defined as the time from the initiation of RT to disease recurrence or progression or death of any cause. OS was defined as the time from the initiation of RT to the date of the last follow-up or the date of death. SPSS software (SPSS Inc., Chicago, IL, USA) was used for statistical analysis, and the Kaplan-Meier method was used to calculate the PFS and OS.

\section{Results}

From March 2003 to June 2017, 40 patients (22 male, 18 female) with high-risk neuroblastoma treated with RT were enrolled in this study. All patients received RT to the primary tumor site. The median age at the time of RT was 4 years (range, 1 to 11 years). The patient characteristics are listed in Table 1. Most of the patients had INSS stage 4 neuroblastoma ( $n=30$ $75 \%$ ). The primary tumor sites were mainly in the abdomen (i.e., 19 adrenal and 14 abdominal). MYCN amplification was 
Table 1. Patients' characteristics $(n=40)$

\begin{tabular}{lc}
\hline \multicolumn{1}{c}{ Characteristic } & Value \\
\hline Age (yr) & $4(1-11)$ \\
Sex & $46.5(19-97)^{\text {a) }}$ \\
Male & $22(55)$ \\
Female & $18(45)$ \\
MYCN status & \\
Amplified & $21(52.5)$ \\
Not amplified & $16(40)$ \\
Unknown & $3(7.5)$ \\
Primary site & $19(47.5)$ \\
Adrenal & $14(35)$ \\
Abdominal & $7(17.5)$ \\
Other site & \\
Initial bone marrow status & $24(60)$ \\
Involved & $16(40)$ \\
Not involved & \\
INSS stage & $1(2.5)$ \\
2 & $9(22.5)$ \\
3 & $30(75)$ \\
4 &
\end{tabular}

Values are presented as median (range) or number (\%). INSS, International Neuroblastoma Staging System.

${ }^{\text {a) }}$ Calculating age in months.

Table 2. Treatment characteristics

\begin{tabular}{lr}
\hline & Value \\
\hline Extent of resection & $36(90)$ \\
Gross total resection & $4(10)$ \\
Subtotal resection & \\
Stem-cell transplantation & $35(87.5)$ \\
Yes & $5(12.5)$ \\
No & \\
Radiation therapy & 3DCRT or IMRT \\
Technique & 14-36/7-20 \\
Dose (Gy/fx)
\end{tabular}

Values are presented as number (\%).

3DCRT, three-dimensional conformal radiation therapy; IMRT, intensity-modulated radiation therapy.

observed in 21 patients (52.5\%).

The treatment characteristics are summarized in Table 2. Thirty-six patients underwent gross total resection (GTR) and 4 patients underwent subtotal resection. After surgery, 35 patients underwent stem-cell transplantation. A total of 40 patients received $\mathrm{RT}$ to the primary tumor site. The median radiation dose was $21 \mathrm{~Gy}$ (range, 14 to $36 \mathrm{~Gy}$ ). The most commonly used RT regimen was $21 \mathrm{~Gy}$ at $1.5 \mathrm{~Gy}$ per fraction

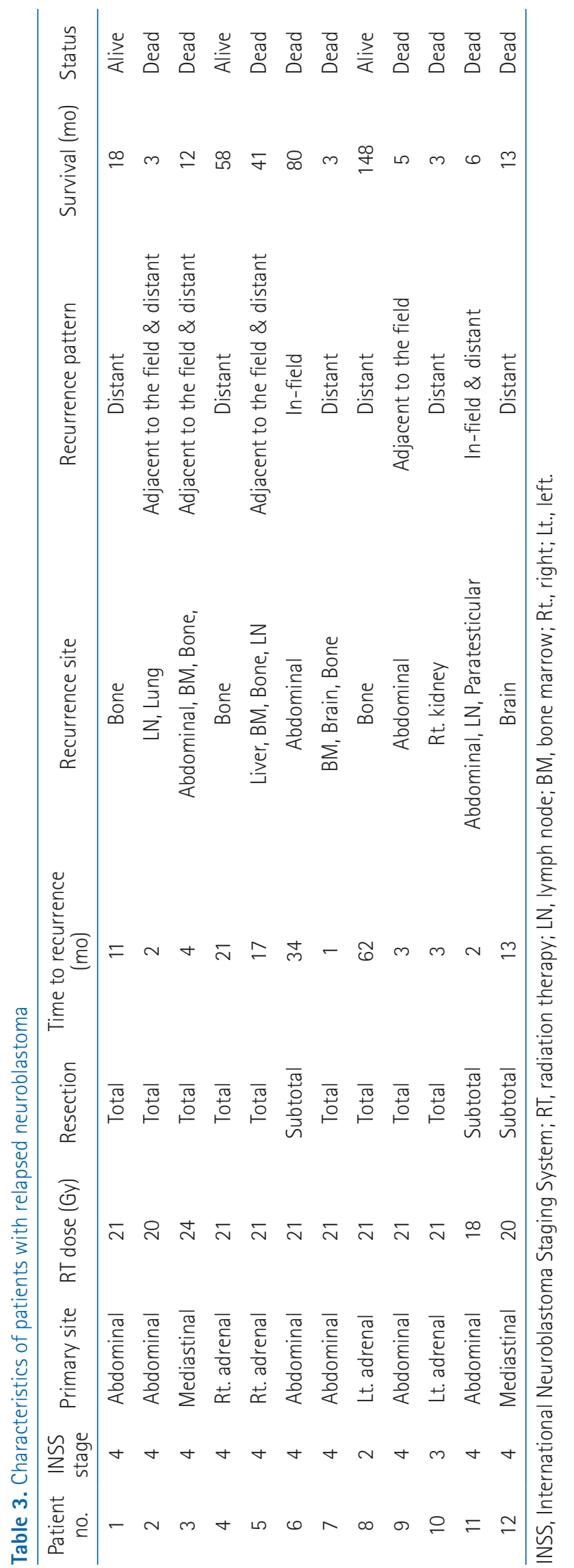

https://doi.org/10.3857/roj.2019.00353 


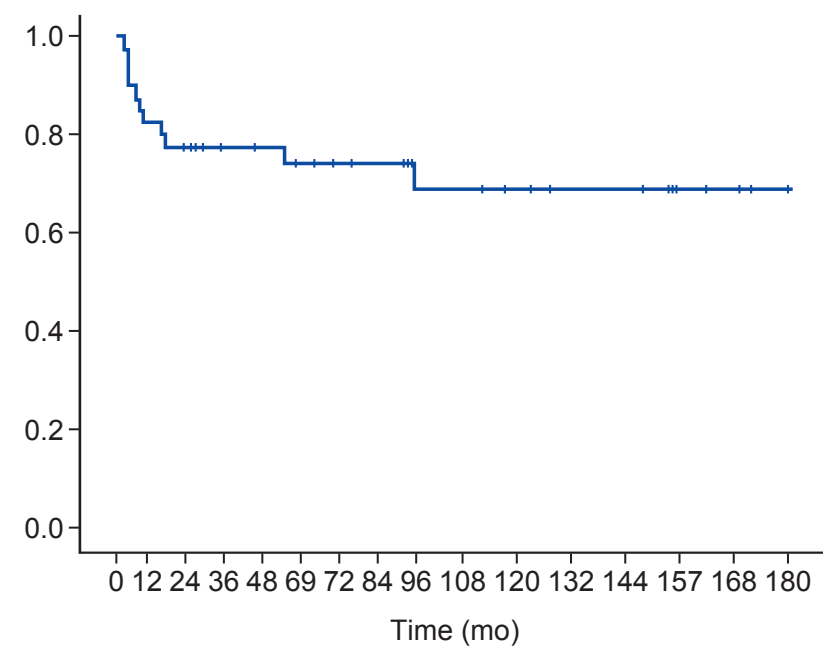

Fig. 2. Overall survival in all patients $(n=40)$.

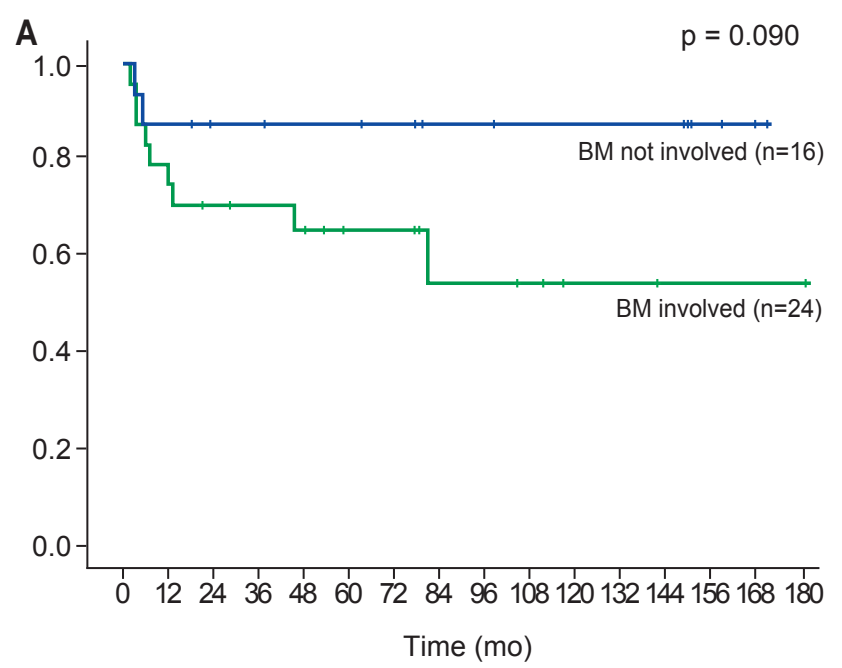

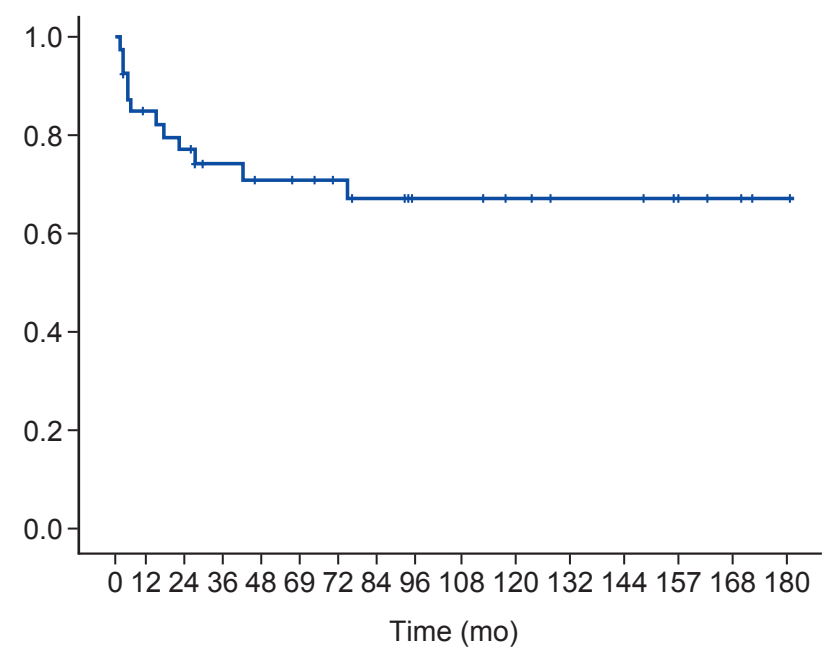

Fig. 3. Progression-free survival of all patients $(n=40)$.

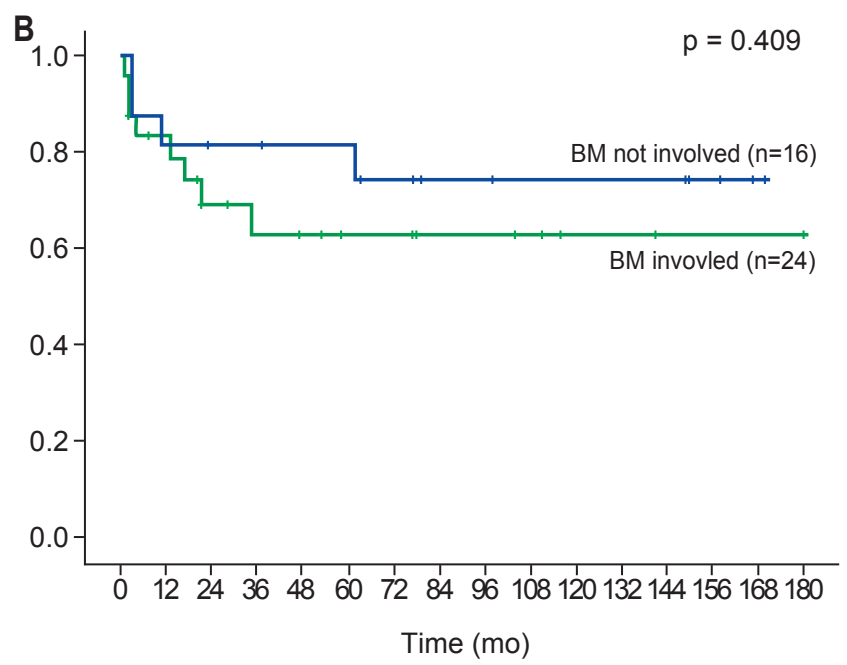

Fig. 4. Overall survival (A) and progression-free survival (B) according to the status of bone marrow (BM) involvement.
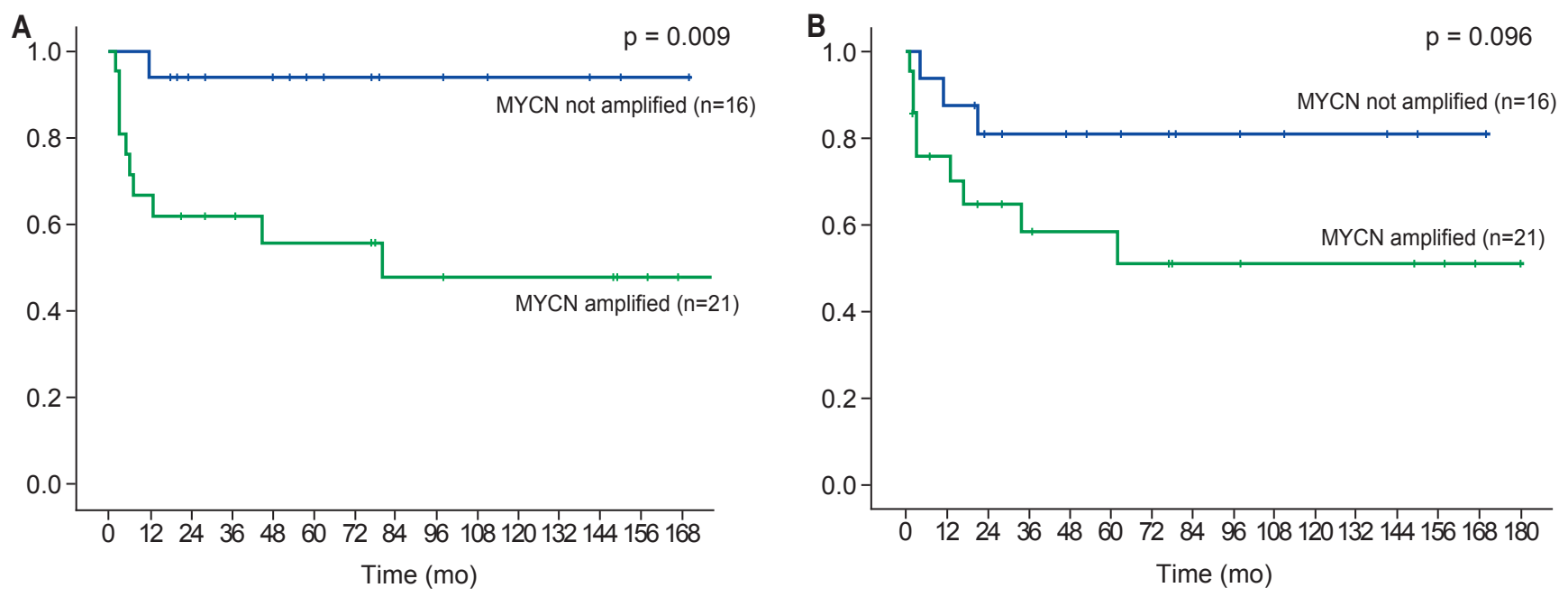

Fig. 5. Overall survival (A) and progression-free survival (B) according to the status of MYCN amplification. 
Table 4. Local control rate of high-risk neuroblastoma in recent studies

\begin{tabular}{lcccc}
\hline \multicolumn{1}{c}{ Study } & Year & No. of patients & Median RT dose (Gy) & Local control rate (\%) \\
\hline Wolden et al. [5] & 2000 & 47 & 21 & 84 at 5 years \\
Kushner et al. [6] & 2001 & 99 & 21 & 89.9 at 3 years \\
Bradfield et al. [7] & 2004 & 21 & 21 & 93 at 2 years \\
Gatcombe et al. [10] & 2009 & 34 & 22 & 94 at 3 years \\
Casey et al. [8] & 2016 & 246 & 21 & 90.2 at 5 years \\
Ferris et al. [9] & 2017 & 67 & 21.6 & 94.0 at 5 years
\end{tabular}

$R T$, radiation therapy.

two times per day (26 patients). In 8 patients, RT was delivered to the primary site and the initial metastatic site at the same time. The decision to treat the metastatic disease was at the discretion of the treating physician. Usually, the decision for administering RT to metastatic sites was based on the presence of persistent disease after induction chemotherapy or if any residual bulky disease remains. At the median followup of 58 months (range, 3 to 180 months), there were 12 cases of recurrence. The individual characteristics of patients with relapsed neuroblastoma and the details of the sites of recurrence are shown in Table 3. Most of the relapsed patients had INSS stage 4 neuroblastoma (10 patients). Among the 12 relapsed patients, 5 had both local and distant recurrence. There were 6 local failure cases and, among these, 4 relapsed adjacent to the radiation field. The other 2 relapsed in the radiation field (i.e., para-aortic and retroperitoneal areas). No disease progression was observed at the irradiated metastatic sites. The main sites of distant metastasis were the bone, lymph nodes, and bone marrow. At the time of analysis, 11 patients had already died (2-80 months after RT).

The 5-year OS was 74.3\% (Fig. 2) and the 5-year PFS was $70.9 \%$ (Fig. 3). There were only 2 cases (5.0\%) of in-field recurrence. OS and PFS tended to be worse in the presence of MYCN amplification or bone marrow involvement. However, there were no statistically significant differences in other results except for OS in patients with MYCN amplification (Figs. $4,5)$.

Most cases of acute toxicities were grade 1, which mainly included nausea, vomiting, and diarrhea. Severe toxicity of RT (>grade 2) was not observed in the current study, although there was a lack of a detailed description of treatment toxicity in the medical records of our hospital.

\section{Discussion and Conclusion}

In patients with high-risk neuroblastoma, local recurrence is the main cause of treatment failure $[14,15]$. Moreover, surgical resection of the primary tumor combined with systemic therapy improves the local control of the disease and the survival of the patient $[15,16]$. Several studies have used RT as a consolidative therapy and have reported successful local control [4-10].

One previous randomized controlled trial compared patients who received RT with those who did not [4]. Patients older than 1 year at the time of the diagnosis of Pediatric Oncology Group stage C neuroblastoma were included in this study. After surgery, the patients received RT to the tumor bed/ residual tumor and regional lymph nodes. The control group received no RT, and all patients were treated with the same chemotherapy regimen. Patients who received RT showed superior treatment outcomes (event-free survival, 59\% vs. 73\%; OS, 32\% vs. 41\%). The results of other studies that showed good local control rates are summarized in Table 4 Bradfield et al. [7] reported a 7\% local failure rate at 2 years from RT. RT was delivered to the primary site and to the initial metastatic sites. Gatcombe et al. [10] reported a 3-year local control rate of $94 \%$. RT was delivered to the primary tumor sites regardless of the resection status and metastatic sites according to chemotherapy response. The median RT dose was $22 \mathrm{~Gy}$, and most common fraction scheme used was 21.6 Gy at $1.8 \mathrm{~Gy} /$ day. Casey et al. [8] reported that the cumulative incidence of local failure at 5 years after RT (1.5 Gy/fraction 2 times a day, 21 Gy in total) was 9.8\% in patients with high-risk neuroblastoma. In this study, all patients received chemotherapy and underwent GTR. Thereafter, the patients received RT to the primary site. Ferris et al. [9] reported that 67 patients with high-risk neuroblastoma received consolidative RT at a median dose of $21 \mathrm{~Gy}$. RT was delivered to patients with high-risk neuroblastoma as part of definitive treatment. All patients were treated with RT to the primary site, and some of them also received $\mathrm{RT}$ to the metastatic sites. The overall local control rate was $92.5 \%$, and no disadvantages were seen 
with respect to pathologic positive nodes, positive surgical margins, or gross residual disease. Although there is only 1 randomized controlled trial, these data may suggest that RT to the primary tumor site is effective for the local control of high-risk neuroblastoma. Further, considering the results of the current study and the other previously published papers, 21-24 Gy is adequate for local disease control in patients with high-risk neuroblastoma.

Although some studies reported that a higher dose is needed to control gross residual tumor after incomplete surgery $[17,18]$, the optimal dose of RT is still unclear. In this study, 2 patients received boost RT for gross residual tumor with a total radiation dose of 27 and $36 \mathrm{~Gy}$, respectively. Local failure was not observed in these patients. Owing to the small number of patients who received boost $R T$, it is difficult to conclude the adequate RT dose. However, given that only patients with residual gross tumor had in-field recurrence and no local failure was observed in patients who underwent boost RT, a higher dose may be effective in treating patients with gross residual tumor. It seems necessary to scrutinize the results of ongoing studies.

In our study, most of the patients underwent GTR (90\%). The local control rate at the irradiated site was 95\%. Only two cases of in-field progression were detected in the current study, and complete resection was not performed in all patients. This result correlates with that of previous reports suggesting the role of RT in improving the local control rate. Given that there is evidence that survival is associated with the local control rate [19], this result suggests that RT plays a vital role in the treatment of high-risk neuroblastoma. Moreover, considering that only patients who had not undergone complete resection experienced recurrence within the RT field, GTR might have an important role in improving local control. This result is consistent with that of a previous study showing favorable outcomes in patients undergoing complete resection [20].

Although metastatic disease is the main cause of death in patients with high-risk neuroblastoma, the role of $\mathrm{RT}$ to metastatic sites is still unclear. Some retrospective studies have demonstrated that RT directed to metastatic sites improves the local control at the irradiated metastatic site [7,21-25]. Casey et al. [21] reported the treatment results of 159 patients who received $\mathrm{RT}$ to metastatic sites as a consolidative therapy. The 5-year local control rate of the treated metastatic site was $81 \%$. Further, the local control rate was better in the treated sites that were cleared with induction chemotherapy. In our study, 8 patients were treated in both the primary site and in the metastatic sites. Of these patients, 4 experienced disease progression, all of whom had an out-of-field recurrence. In the other 4 patients without recurrence, long-term survival was achieved (98-167 months). There was no case of disease progression within the RT field. Currently, data supporting the efficacy of RT to the metastatic site are insufficient; however, considering that recurrence is common in previously involved sites in patients with neuroblastoma [13,23,24], RT to metastatic sites may improve the tumor control and may be effective in preventing disease progression and prolonging the long-term disease control. RT to the metastatic sites may therefore be beneficial in the treatment of patients with distant tumor involvement. Additional studies are needed to clarify whether RT to metastatic sites is beneficial.

In the older treatment protocol, total body irradiation (TBI) was used as part of a consolidative treatment with chemotherapy and bone marrow transplantation $[13,26]$ Although TBI was excluded from the treatment protocol because of its long-term toxicity, some articles reported the efficacy of TBI $[22,25]$. In our study, 2 patients received TBI. It is important to analyze the treatment outcome according to $\mathrm{TBI}$ however, it is difficult to evaluate the role of TBI because of the small number of patients who received TBI in our patient cohort.

The current study has several limitations, including its retrospective nature. First, the composition of the enrolled patients is not uniform. Most of the patients enrolled in this study had INSS stage 4 neuroblastoma with distant involvements. Although these patients are included in a single group (stage 4), they may have different prognoses based on the location of the metastatic disease. A further limitation is the heterogeneity of the chemotherapy protocols. Most of the chemotherapy regimens were based on COGANBLOOP1 and CCG3891, although other protocols were included in the patient cohort, with diverse differences in the chemotherapy regimen for patients with recurrence. In addition, only a small number of patients were analyzed, thus leading to insufficient statistical power. However, owing to the rarity of the disease, it would be difficult to conduct a large study. The current study presents the treatment outcome of RT in patients with highrisk neuroblastoma in a single hospital, where the treatment was provided by the same medical team using a relatively homogeneous protocol.

In conclusion, RT seems to be adequate for disease control in patients with high-risk neuroblastoma. RT directed to the primary tumor site provides good local control. However, considering that metastatic disease is an important factor 
that worsens the prognosis of patients with high-risk neuroblastoma, preventing distant recurrence is essential to improve the treatment outcome. It may be effective to irradiate the metastatic sites; however, supporting evidence is still lacking. Therefore, additional studies are needed to clarify whether RT to metastatic sites is adequate for treating patients with high-risk neuroblastoma.

\section{Conflict of Interest}

No potential conflict of interest relevant to this article was reported.

\section{References}

1. Gurney JG, Ross JA, Wall DA, Bleyer WA, Severson RK, Robison LL. Infant cancer in the U.S.: histology-specific incidence and trends, 1973 to 1992. J Pediatr Hematol Oncol 1997;19:42832.

2. Laverdiere C, Liu Q, Yasui Y, et al. Long-term outcomes in survivors of neuroblastoma: a report from the Childhood Cancer Survivor Study. J Natl Cancer Inst 2009;101:1131-40.

3. Woods WG, Tuchman $M$, Bernstein $M L$, et al. Screening for neuroblastoma in North America: 2-year results from the Quebec Project. Am J Pediatr Hematol Oncol 1992;14:312-9.

4. Castleberry RP, Kun LE, Shuster JJ, et al. Radiotherapy improves the outlook for patients older than 1 year with Pediatric Oncology Group stage C neuroblastoma. J Clin Oncol 1991;9:789-95

5. Wolden SL, Gollamudi SV, Kushner BH, et al. Local control with multimodality therapy for stage 4 neuroblastoma. Int J Radiat Oncol Biol Phys 2000;46:969-74.

6. Kushner BH, Wolden S, LaQuaglia MP, et al. Hyperfractionated low-dose radiotherapy for high-risk neuroblastoma after intensive chemotherapy and surgery. J Clin Oncol 2001;19:2821-8.

7. Bradfield SM, Douglas JG, Hawkins DS, Sanders JE, Park JR. Fractionated low-dose radiotherapy after myeloablative stem cell transplantation for local control in patients with high-risk neuroblastoma. Cancer 2004;100:1268-75.

8. Casey DL, Kushner BH, Cheung NK, Modak S, LaQuaglia MP, Wolden SL. Local control with 21-Gy radiation therapy for high-risk neuroblastoma. Int J Radiat Oncol Biol Phys 2016;96:393-400.

9. Ferris MJ, Danish H, Switchenko JM, et al. Favorable local control from consolidative radiation therapy in high-risk neuroblastoma despite gross residual disease, positive margins, or nodal involvement. Int J Radiat Oncol Biol Phys 2017;97:806-12.

10. Gatcombe HG, Marcus RB Jr, Katzenstein HM, Tighiouart M, Esiashvili N. Excellent local control from radiation therapy for high-risk neuroblastoma. Int J Radiat Oncol Biol Phys 2009;74:1549-54.

11. Brodeur GM, Pritchard J, Berthold F, et al. Revisions of the international criteria for neuroblastoma diagnosis, staging, and response to treatment. J Clin Oncol 1993;11:1466-77.

12. Seif $A E$, Naranjo $A, B a k e r ~ D L$, et al. A pilot study of tandem high-dose chemotherapy with stem cell rescue as consolidation for high-risk neuroblastoma: Children's Oncology Group study ANBLOOP1. Bone Marrow Transplant 2013;48:947-52

13. Matthay KK, Villablanca JG, Seeger RC, et al. Treatment of high-risk neuroblastoma with intensive chemotherapy, radiotherapy, autologous bone marrow transplantation, and 13-cis-retinoic acid. N Engl J Med 1999;341:1165-73.

14. Halperin EC. Long-term results of therapy for stage $C$ neuroblastoma. J Surg Oncol 1996;63:172-8.

15. Matthay KK, Atkinson JB, Stram DO, Selch $M$, Reynolds $C P_{1}$ Seeger RC. Patterns of relapse after autologous purged bone marrow transplantation for neuroblastoma: a Children's Cancer Group pilot study. J Clin Oncol 1993;11:2226-33.

16. Haase GM, O'Leary MC, Ramsay NK, et al. Aggressive surgery combined with intensive chemotherapy improves survival in poor-risk neuroblastoma. J Pediatr Surg 1991;26:1119-24.

17. Casey DL, Kushner BH, Cheung NV, Modak S, LaQuaglia MP, Wolden SL. Dose-escalation is needed for gross disease in high-risk neuroblastoma. Pediatr Blood Cancer 2018;65:e27009.

18. Haas-Kogan DA, Swift PS, Selch $M$, et al. Impact of radiotherapy for high-risk neuroblastoma: a Children's Cancer Group study. Int J Radiat Oncol Biol Phys 2003;56:28-39.

19. Pai Panandiker AS, McGregor L, Krasin MJ, Wu S, Xiong $X$, Merchant TE. Locoregional tumor progression after radiation therapy influences overall survival in pediatric patients with neuroblastoma. Int J Radiat Oncol Biol Phys 2010;76:1161-5.

20. LaQuaglia MP, Kushner BH, Heller G, Bonilla MA, Lindsley $K L$, Cheung NK. Stage 4 neuroblastoma diagnosed at more than 1 year of age: gross total resection and clinical outcome. J Pediatr Surg 1994;29:1162-6.

21. Casey DL, Pitter KL, Kushner BH, et al. Radiation therapy to sites of metastatic disease as part of consolidation in highrisk neuroblastoma: can long-term control be achieved? Int J Radiat Oncol Biol Phys 2018;100:1204-9.

22. Li R, Polishchuk $A$, DuBois $S$, et al. Patterns of relapse in high- 
risk neuroblastoma patients treated with and without total body irradiation. Int J Radiat Oncol Biol Phys 2017;97:270-7.

23. Mazloom A, Louis CU, Nuchtern J, et al. Radiation therapy to the primary and postinduction chemotherapy MIBG-avid sites in high-risk neuroblastoma. Int J Radiat Oncol Biol Phys 2014;90:858-62.

24. Polishchuk AL, Li R, Hill-Kayser $C$, et al. Likelihood of bone recurrence in prior sites of metastasis in patients with highrisk neuroblastoma. Int J Radiat Oncol Biol Phys 2014;89:83945.
25. Sibley GS, Mundt AJ, Goldman S, et al. Patterns of failure following total body irradiation and bone marrow transplantation with or without a radiotherapy boost for advanced neuroblastoma. Int J Radiat Oncol Biol Phys 1995;32:1127-35.

26. George RE, Li S, Medeiros-Nancarrow C, et al. High-risk neuroblastoma treated with tandem autologous peripheralblood stem cell-supported transplantation: long-term survival update. J Clin Oncol 2006;24:2891-6. 